\section{Potassium Dioxalato Diaquo Chromium (III) Complexes: Trans-Cis Isomerization}

The X-ray diffraction data of van Niekerk and Schoening ${ }^{1}$, obtained from a sample of red potassium chromi oxalate prepared in these laboratories and corresponding analytically to $\mathrm{K}\left[\mathrm{Cr}\left(\mathrm{H}_{2} \mathrm{O}\right)_{2}\left(\mathrm{C}_{2} \mathrm{O}_{4}\right)_{2}\right] \cdot 3 \mathrm{H}_{2} \mathrm{O}$, led these workers to affirm that this salt has the trans configuration, as deduced by $W_{\text {erner }}{ }^{2}$ from other evidence. We have investigated the colour and other changes which occur when aqueous solutions of this salt are aged under different conditions, and our results show that the changes are due to transcis isomerization, uncomplicated by olation phenomena. Since the same ultimate molar extinction coefficient is attained over a wide range of temperature and concentration, we conclude that the transformation to the cis configuration proceeds to practical completion. Kinetically, the reaction obeys a firstorder law, and the dependence of the observed velocity constant upon temperature is found to be adequately described by the Arrhenius equation, where $A=3 \times 10^{9}$ and $E=-17.9 \mathrm{k} . c a l . / m o l$. (see graph). Confirmation of the practical completion of the trans-cis conversion was obtained by isolation of the blue cis salt, $\mathrm{K}\left[\mathrm{Cr}\left(\mathrm{H}_{2} \mathrm{O}\right)_{2}\left(\mathrm{C}_{2} \mathrm{O}_{4}\right)_{2}\right] .2 \mathrm{H}_{2} \mathrm{O}$, moderately dilute solutions of which are optically stable when olation is excluded.

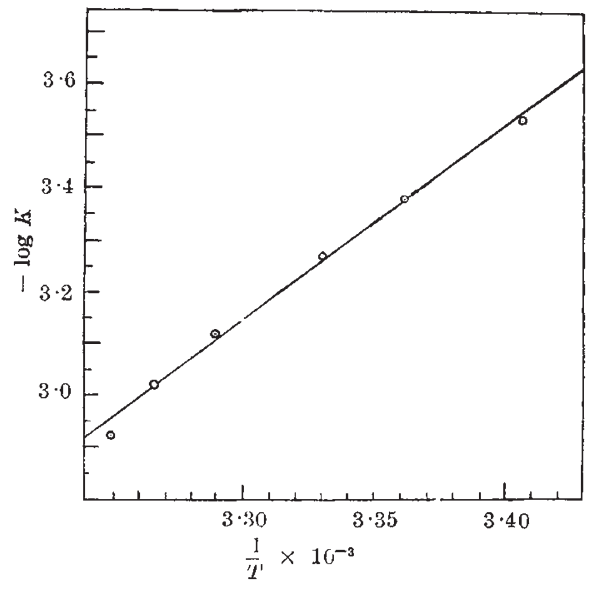

For our investigations we employed the potassium trans salt prepared by Croft's method ${ }^{3}$ and purified by digesting in $\mathbf{l}$ per cent nitric acid (to destroy traces of potassium trioxalato chromiate) prior to recrystallization. 'The corresponding cis salt was prepared from the above by digesting $40 \mathrm{gm}$. with $100 \mathrm{ml}$. water on the steam bath for $1 \mathrm{hr}$., cooling rapidly, and filtering off the sludge which separated after a short time. The latter, after washing with alcohol and ether and drying over sulphuric acid, gave rise to a fine lilac-coloured powder having properties identical with those of the cis salt described by Werner ${ }^{2}$. The absorption curve of a $0.01-M$ solution of this product was found to be practically identical over the range $300-740 \mathrm{~m} \mu$ with that of the mother liquor appropriately diluted, although slow cooling of the undiluted mother liquor results in the deposition of crystals of the trans salt over a period of days.

These results show that solutions of relatively pure potassium cis dioxalato diaquo chromium (III) can be prepared conveniently from the more readily purified trans salt simply by warming solutions of the latter for a few hours before diluting to the

\begin{tabular}{|c|c|c|c|c|}
\hline \multirow{2}{*}{$\begin{array}{cc}\text { Complex ion } \\
{\left[\mathrm{Cr}\left(\mathrm{H}_{2} \mathrm{O}\right)_{2}\left(\mathrm{C}_{2} \mathrm{O}_{4}\right)_{2}\right]^{-}}\end{array}$} & \multicolumn{2}{|c|}{ Optical density } & \multicolumn{2}{|c|}{$\begin{array}{c}\text { Titration data } \\
\text { Titrat }\end{array}$} \\
\hline & $\operatorname{Max} .(\mathrm{m} \mu)$ & $\varepsilon$ (molar) & & \\
\hline trans & 416 & $34 \cdot 4$ & $7 \cdot 5$ & $10 \cdot 5$ \\
\hline cis & $\begin{array}{l}555 \\
416 \\
562\end{array}$ & $\begin{array}{l}32 \cdot 0 \\
68.5 \\
51 \cdot 0\end{array}$ & $7 \cdot 5$ & $9 \cdot 7$ \\
\hline
\end{tabular}

desired concentration, isolation of the solid being scarcely necessary for many purposes.

The molar extinction coefficients at the two optical density maxima on the absorption curves are given in the accompanying table and were determined with the Beckman DU spectrophotometer. In the case of the trans salt the observations were made in stages, using freshly prepared $0.01-M$ solutions at $8^{\circ} \mathrm{C}$. for each stage of the absorption curve, in order to minimize drift errors; but this was not necessary for the cis salt, which was optically stable even at elevated temperatures.

The titration constants shown in the table are calculated from titration curves obtained at $8^{\circ} \mathrm{C}$. in the same way, using a Cambridge $p H$-meter and glass electrode assembly, the curves being corrected for blank potassium hydroxide titrations carried out on conductivity water under the same conditions of ionic strength and temperature. These 'constants' refer to the acidic dissociation of the water molecules bound to the complex, and are uncorrected for the ionic strength of the solutions. An exact calculation of the relative contribution of the 'electrostatic' and 'rest' effects to the difference $p K_{2}-p K_{1}$, according to the principles discussed by Bjerrum ${ }^{4}$, is therefore scarcely justified at this stage. Nevertheless, since $n$ is larger for the trans ion than for the cis isomer, whereas the reverse would hold were the electrostatic effect the principal factor involved, it is clear that considerable differences in electron distribution must exist between the two isomeric forms of the complex ion.

Further investigations are proceeding, and it is hoped to publish a full account elsewhere in due course.

\section{G. E. CUNNINGHam}

R. W. BURLEY

M. 'T. FRIEND

Leather Industries Rescarch Institute, Rhodes University,

Grahamstown, South Africa. Jan. 11.

${ }^{1}$ van Niekerk, J. N., and Schoening, F. R. I., Nature, 166, 108 (1950); Acta Cryst., 4, 35 (1951).

${ }^{2}$ Werner, A., Ann. Phys., 406, 261 (1914).

${ }^{3}$ Croft, H., Phil. Mag., 21, 197 (1842).

4 Bjerrum, N., Z. phys. Chem., 106, 219 (1923); Svensh. Kem. Tid. 28, 2 (1926).

\section{Sugar Chromatography of Mammary Gland Extracts}

THE communication from Drs. Caputto and Trucco" on "A New Galactose-containing Compound from Marnmary Gland" interests us because our own work on lactose synthesis did at one stage give results very similar to those they now report. The interpretation we put upon our results, however, was different from that which they derive from theirs; further, the tentative conclusions we are now tempted to draw from some of our more recent work are also in conflict with the views expressed in their letter.

Our earlier report (see Malpress ${ }^{2}$ ) showed the presence of a fraction obtained by the direct trichlor- 